Revista de la red interuniversitaria de estudios sobre las literaturas rioplatenses contemporáneas en Francia

$20 \mid 2019$

Situación

\title{
Poesía parenética y guerra: análisis de seis poemas de Vicente Luy
}

Poésie parénétique et guerre: analyse de six poèmes de Vicente Luy

Parenetic poetry and war: analysis of six poems by Vicente Luy

\section{Fernando Bogado}

\section{(2) OpenEdition}

Journals

Electronic version

URL: https://journals.openedition.org/lirico/8732

DOI: $10.4000 /$ lirico.8732

ISSN: 2262-8339

\section{Publisher}

Réseau interuniversitaire d'étude des littératures contemporaines du Río de la Plata

Electronic reference

Fernando Bogado, «Poesía parenética y guerra: análisis de seis poemas de Vicente Luy», Cuadernos LIRICO [En línea], 20 | 2019, Publicado el 02 julio 2019, consultado el 08 junio 2021. URL: http:// journals.openedition.org/lirico/8732 ; DOl: https://doi.org/10.4000/lirico.8732

This text was automatically generated on 8 June 2021.

\section{(c) $(1)$}

Cuadernos LIRICO está distribuido bajo una Licencia Creative Commons Atribución-NoComercial-

SinDerivar 4.0 Internacional. 


\title{
Poesía parenética y guerra: análisis de seis poemas de Vicente Luy
}

\author{
Poésie parénétique et guerre: analyse de six poèmes de Vicente Luy \\ Parenetic poetry and war: analysis of six poems by Vicente Luy
}

\section{Fernando Bogado}

\section{"San Luy"}

El poeta es un dios caído, cuyo sufrimiento
proclama su título imprescindible. (Benichou

2017: 600)

1 La editorial Elemento Disruptivo, que entra dentro de la compleja categoría de "editoriales independientes" ${ }^{1}$ con la cual el mercado editorial se ha manejado en los últimos años (o, más específicamente, desde 2001 en adelante), sacó una colección de "estampitas" con la imagen de diferentes poetas rápidamente reconocibles dentro del mundo (o mundillo) de la poesía argentina contemporánea. En la colección se puede encontrar a Idea Vilariño, a José Sbarra, y también, desde hace poco tiempo, a Vicente Federico Luy (1961-2012), poeta nacido en Córdoba que se ha impuesto, en el mismo período de tiempo, como uno de los nombres insoslayables de la poesía "underground", "emergente", "alternativa" local. El hecho mismo de que haya aparecido en una estampita no marca otra cosa que la progresiva entrada del nombre de Luy dentro del espectro de autores que deben ser leídos, o cuyo estilo empieza ya a imitarse, a propagarse, bajo la lógica de un esperable contagio de ciertas formas literarias en la producción de los poetas más jóvenes. Además, su temprana muerte, ocasionada por un trágico suicidio, en febrero de 2012, no ha hecho otra cosa que entronizarlo en ese margen de lectura como un "poeta maldito". Perspectiva "malditista" que tiñe la recepción de toda su producción: la progresiva reedición de sus libros, la proyección de una venidera biografía sobre su ya de por sí rica (y difícil) vida ${ }^{2}$ y también, claro, su entrada en el escueto canon de los poetas que hay que leer como material de estudio para los cursos de análisis de poesía. 
2 ¿Quién fue Vicente Luy? Se hace imprescindible la mención de un autor que recién está comenzando a ser leído con cierto interés por parte de diferentes críticos. Vicente Luy nació en Córdoba, Argentina, hijo de Lucienne Larrea y de Gilbert Luy, un empresario francés vinculado a la fábrica de automóviles Renault. Lucienne, o Luciana, y Gilbert mueren en noviembre de 1961, apenas meses nacido Vicente, en un accidente aéreo en San Pablo, Brasil. A partir de ese momento, el responsable de la crianza de Vicente es su abuelo, el poeta español Juan Larrea, perteneciente a la Generación del '27 española, amigo íntimo de César Vallejo, pieza fundamental del mundo intelectual ibérico que huyó hacia América por las obvias consecuencias del fin de la Guerra Civil. Luego de pasar por Perú, y en el medio de una diáspora cuyo cierre no se advertía, terminó en Córdoba, trabajando en la universidad de la capital. Allí fundó el espacio de estudio de la poesía de su amigo peruano, la conocida "Aula Vallejo".

3 Fallecido su abuelo, el 9 de julio de 1980, con Vicente atravesando el Servicio Militar Obligatorio, comienza luego un derrotero un tanto más personal de un poeta en formación, que publica su primer libro en 1991, bajo el título de Caricatura de un enfermo de amor. Luego de ese libro, el otro gran mojón en la entrada de Luy dentro del escenario poético es su activa participación en el grupo de músicos y poetas bautizado con el nombre de "Verbonautas", en donde también se encontraban Osvaldo Vigna, Hernán (poeta que firma sin apellido), Eduardo Nocera, Karina Cohen, Palo Pandolfo, entre otros. Formados a partir de un grupo anterior, denominado "Comando Poético Literario", los "Verbonautas" aparecieron en la escena under porteña en diciembre de 1995, con un proyecto de intervención estética a partir de encuentros en diferentes bares, a título de recitales de poesía, y "atentados poéticos", acciones que consistían en recitar de improviso poemas en espacios públicos (generalmente, bares y cafés). En 1999, los miembros del grupo sacaron la única antología firmada por el colectivo, Verbonautas. Acción poética, por el sello Eudeba, a partir de sus conexiones con el Centro Cultural Ricardo Rojas. En el mismo año, Luy sacaría su segundo libro, uno de los más importantes de su producción, aparecido en papel ilustración, con dibujos y una tapa dura tan llamativa y provocativa como su título, La vida en Córdoba.

El segmento más importante de la producción de Luy se inicia con ese libro de 1999 y culmina con el único texto aparecido de manera póstuma, hasta el momento. Los libros posteriores a La vida en Córdoba mezclaron, en igual medida, cierto intento de antología personal y una marcada tendencia a intervenir a partir de las poéticas de sus compañeros (como se ve en el libro Poesía moderna y en la versión final de ese proyecto, Aviones, compuesto en parte por poemas de otros autores sin firma, publicación que lo llevó a tener problemas con los responsables de los textos antologados; o ¡Qué campo ni campo!, en donde directamente abre espacios del libro para la aparición de poemas de sus amigos más cercanos, Osvaldo Vigna y Hernán). El póstumo que cierra esta breve serie es Plan de operaciones / La única manera de vivir a gusto es estando poseído, de 2014. Dos libros en uno, estrictamente. El primero corresponde al último que Luy armó con Hernán como diseñador (rol que ocuparía en diversas publicaciones de Vicente); el segundo es un libro construido a partir de poemas inéditos, fragmentos y correos electrónicos que fue enviando a diferentes contactos en el último tramo de su vida. Como agrega Hernán en el prólogo del citado ejemplar, "El 23 de febrero [de 2012] Vicente saltó al vacío desde un séptimo piso en la ciudad de Salta" (2014: 10).

5 Paul Benichou, en el último tomo de su historia de la constitución en el campo intelectual francés de la figura de escritor, La escuela del desencanto, considera que el 
tono derrotista y la progresiva separación del poeta del ámbito social (que acompaña con un distanciamiento de lo escrito con respecto a la inmediata comprensión del gran público) es el resultado de un compromiso político frustrado por las revoluciones de 1830 y, sobre todo, de 1848 (y el posterior Golpe de Estado de Luis Bonaparte en 1851). Esa decepción con respecto a la coyuntura impregnó formalmente sus trabajos con un tono de difícil comprensión, que los simbolistas del último cuarto del siglo XIX transformarían en una estética bien definida. Pero Benichou señala, una y otra vez, en su estudio de la segunda generación romántica francesa (Nerval, Gautier, entre otros) que el simbolismo, o la idea misma de "malditismo" que empieza a operar con fuerza en los escritores ligeramente posteriores, es el resultado de una decepción de tipo sociopolítico que atraviesa las disposiciones formales de sus producciones. Anota Benichou: "El poeta maldito, entre un ideal avaro de comunicación y un auditorio sordo, vive en el fracaso; sin embargo, es soberano en su soledad" (2017: 601). Las formas actuales de construcción del "poeta maldito" obvian o fetichizan irreflexivamente la relación del poeta con su coyuntura. Luy vivió y escribió durante el "menemato" y el kirchnerismo, dos momentos políticos que recuperó en sus propios poemas (sobre todo, en lo que corresponde a claras referencias temáticas al menemismo), pero ni siquiera la mención explícita soluciona las contradicciones inherentes a varios análisis de poesía que avanzan sobre su escritura. No mencionar esas instancias de coyuntura, o no poner en evidencia las relaciones que esa producción poética mantiene con su presente, es tan problemático, metodológicamente, como llevar adelante un análisis meramente descriptivo que no arriesgue una interpretación por temor a incurrir en la falsa creencia de la transparencia de la forma literaria con respecto a su contexto. Ni la lectura temática y representativa de la poesía, ni la descripción formal, son extremos lícitos para un auténtico estudio. En el presente trabajo, llevaremos adelante un análisis de tipo inmanente que tratará de poner en evidencia operaciones literarias que, sólo a través de su particularidad, buscan intervenir en el contexto, antes que "representarlo". Theodor Adorno, en Teoría estética, establece en algunos párrafos claves la metodología de tipo inmanente-dialéctica que habilita a una lectura de las complejas mediaciones que cualquier producción estética, incluyendo la poesía, mantiene en su relación con la sociedad:

En la obra de arte, y en la teoría de la misma, por tanto, sujeto y objeto son sus momentos propios y su dialéctica se da en aquello de que la obra de arte compone: material, expresión, forma, aunque estos componentes estén duplicados. Los materiales llevan la marca de quienes los emplearon para hacer la obra de arte; la expresión objetiva en la obra y en sí misma, lo es en cuanto movimiento subjetivo; la forma ha de madurar subjetivamente según las necesidades del objeto si no quiere tener con lo conformado una relación puramente mecánica. De forma análoga a la constitución de algo dado en la teoría del conocimiento, lo que aparece como objetivamente impenetrable ante el artista, como sucede muchas veces con los materiales, no es sino sujeto sedimentado; lo que es en apariencia lo más subjetivo, la expresión, es también objetivo porque la obra se elabora desde ella y la incorpora a sí misma; es finalmente una conducta subjetiva en que se imprime la objetividad (1984: 220).

6 La subjetividad, entendida como un escorzo más de la composición estética, es un momento de la dialéctica sujeto-objeto formada en la obra de arte. El título de "poeta maldito", su propia biografía, su modo de vivir el tiempo que le tocó vivir a Vicente Luy, puede ser recuperado como un elemento elocuente para el análisis siempre que se lo considere como parte de este ir y venir permanente entre lo subjetivo y lo objetivo de la obra, sin síntesis que inmovilice. Lo pertinente, ahora, es concentrarse en los 
recursos de los poemas para poder luego relevar los modos de intervención en su contexto y, aún, en el presente.

\section{Hablarle al pueblo: frase rectora y estructura en espejo}

7 De la colección completa de poemas de la obra de Luy, nuestro corpus se centrará en los textos presentados bajo la frase rectora "Vicente habla al pueblo", presentes en Poesía moderna (2000) / Aviones (2002), pero recopilados en la antología La sexualidad de Gabriela Sabatini (2006), el libro llamado precisamente Vicente habla al pueblo (2007) y Poesía popular argentina (2009). La obra de Luy, a partir de La sexualidad de Gabriela Sabatini, se convierte en una suerte de ejercicio de reescritura y recopilación permanente: Luy vuelve sobre poemas ya publicados, los recupera para nuevas antologías o los reescribe para nuevas ediciones, como si fuese preciso volver a presentar lo que ya estaba trabajado en un libro anterior. Como si determinados poemas todavía fueran elocuentes para el contexto en el cual un nuevo libro aparecía. Esto también habla de dos condiciones pertinentes a la hora de retomar a la figura "biográfica" de Luy: su estado de salud, el cual fue material de más de un poema y que cerró de manera trágica con su suicidio; pero, también, la idea de una obra consistente, que debía perfeccionarse, pulirse con el paso del tiempo. La reescritura es uno de los procedimientos más importantes en la obra de Luy, algo que se hace evidente, sobre todo, en libros cronológicamente contiguos: en Poesía popular argentina hay una sección de borradores de poemas que luego aparecerán con otra forma (no se puede decir "definitiva") en Plan de operaciones / La única manera de vivir a gusto es estando poseído. Lo mismo pasa con los fragmentos de poemas que están en No le pidan peras a Cúper y que en las antologías posteriores (sobre todo, en Poesía popular argentina) aparecen como poemas "terminados". La instancia "borrador", en una producción poética que vuelve siempre sobre lo ya escrito, es un término que puede muy bien aplicarse a la totalidad de la obra: un inmenso borrador de un poema imposible, pero que al mismo tiempo no se proyecta con su posición "trascendente" a los poemas presentes. Recuperando la terminología crítica que Deleuze y Guattari re-utilizan del campo del psicoanálisis lacaniano, no hay un objeto definitivo y perdido, ni siquiera operando en un "más allá" que sirve de horizonte para la multiplicación de objetos "a la mano" investidos, sino objetos petit $a$, una proliferación de fragmentos de algo que podría estar como no, pero que no condiciona esa proliferación ${ }^{3}$. No hay poemas pensados como fragmentos de un poema mayor, sino poemas fragmentarios que circulan, regresan, se reacomodan en nuevas series, se transforman ${ }^{4}$.

8 Los poemas de la serie "Vicente habla al pueblo", que aparecen en diversos libros, pero que recortaremos de los reunidos en Poesía popular argentina por ser la última edición donde se presentaron, poseen características sobre las que me concentraré para constituir el objeto de estudio de este artículo, que consistiría en los modos de articulación inmanente de la poesía de Luy en relación a su contexto de aparición. En primer lugar, el poema es parte de un ensamblaje en donde texto e imagen dialogan: las ilustraciones de María Angélica Vaca Narvaja se encuentran acompañando cada parte textual, siendo todas retratos con espíritu de boceto de Luy sentado, de perfil, mirando hacia la derecha. La única excepción es el poema "Vicente habla al pueblo VIII" (2009: 102, cfr. anexo V), sin ilustración, cuya numeración no corresponde a ninguna serie 
explícita en Poesía popular argentina. En los poemas con ilustraciones, el escorzo textual puede encontrarse arriba, abajo o a la derecha, siempre opuesto a la ilustración. Esa disposición da la idea de una estructura en espejo: son dos partes de lo mismo, aunque de diferente factura (ilustración contra texto), opuestas, pero componiendo la misma pieza. El movimiento que la puesta en serie de estos poemas presenta tampoco es de desmerecer: la colocación de lo textual y de la ilustración no es estática, sino que se presenta con cierto movimiento, aunque limitado, ya que no hay relación con las diagonales, ni creación de efectos de perspectiva, ni superposición de escorzos. Lo cual refuerza la disposición en espejo. La idea de una compleja unidad también se sostiene sobre el hecho de que el fondo es siempre de un color pleno, gris o negro, sobre el que la imagen o el texto se disponen $u$ oponen (como el caso del poema "Cuando vos agarrás, por ej...", cfr. anexo IV, en donde el texto se encuentra sobre un fondo blanco, la página misma, y la ilustración sobre un fondo negro, con la presencia particular de la onomatopeya propia del sueño, tres letras "Z" que nos remiten a una ilustración "dormida", algo que puede presuponerse por su disposición en horizontal).

La segunda característica es la frase rectora "Vicente habla al pueblo". Me parece pertinente la idea de este nombre en particular, "frase rectora", ya que "título" supondría una ubicación prototípica del sintagma en la parte superior, sobre el margen izquierdo, en negrita, entrecomillado o subrayado. Y este no es el caso. La primera aparición de la frase rectora en el poema "Controle el pueblo sus ingresos..." (2009: 30, cfr. anexo I), por ejemplo, se da en la parte inferior, en el pie de la página, en un cuerpo menor con respecto al resto del escorzo textual. Además, en el resto de los poemas de la serie, leemos variaciones de esa frase rectora: "Vicente habla al pueblo (ahora en verso)" (44, cfr. anexo II); "Vicente asume su necesidad de amor" (61, cfr. anexo III); "Vicente sueña con Lanata y le pregunta (cuál es el fin último es la pregunta)" $(65, c f r$. anexo IV); el comentado "Vicente habla al pueblo VIII" (102, cfr. anexo V) y, finalmente, "Vicente vuelve a hablar al pueblo" (125, cfr. anexo VI), en la sección del libro dedicada a "Fragmentos y más poemas". La frase rige sobre la lectura del poema, estableciendo una indicación acerca de cómo debe ser leído, cómo debe ser interpretado. Allí, se restringe la supuesta indeterminación de la identidad del lector: el interlocutor válido es siempre nombrado por el poema, "pueblo", y cada lector individual ocupa, al menos, provisoriamente, la posición de "parte del pueblo" en la medida en que "lee" el poema.

La tercera característica corresponde a las temáticas abordadas en cada poema. Si bien hablar de "tema", en la medida en que es una categoría semántica, siempre es complejo a la hora de analizar un poema; en este caso, debido a la evidente pregnancia de ciertos tópicos, se puede establecer un conjunto de recurrencias que permiten vincular entre sí a cada uno de los ensamblajes de texto e imagen -a los que bien se puede llamar composiciones-. En los seis poemas, la interpelación del yo lírico (categoría que, por el momento, será de utilidad) al interlocutor "pueblo", construido desde la frase rectora, siempre funciona como un intento de indicación acerca de algún tipo de actividad concreta a realizar en función de un problema o asunto de carácter público, propio del orden del Estado y su administración, vigilancia o incluso como parte de un discurso acerca de su naturaleza misma. El esperable entramado de tema y forma obliga a la recuperación de la distinción de Roman Jakobson acerca de las funciones del lenguaje: la presencia de una función apelativa acompañando la función poética deriva en la aparición de la forma verbal de imperativo. El primer poema de la serie (cfr. anexo I) empieza, precisamente, con una forma semejante: "Controle el pueblo sus ingresos..." (subrayado propio). Luego, tenemos en el segundo poema "Remítase a la consciencia / 
oblíguese a la memoria / esfuércese el buen señor..." (subrayado propio). El resto de los poemas operan con formas verbales en presente del modo indicativo, cambiando la función apelativa por un tipo de constatación (la fuerte presencia del verbo "es"); o increpando a un interlocutor diferente, pero con el mismo objetivo de apuntar a la "cosa pública" (el poema en donde la frase rectora tiene un nombre propio cuya referencia es posible identificar: "Lanata" ${ }^{5}, c f r$. anexo IV); o, finalmente, disponiendo de una estructura condicional que vuelve a aparecer en un conocido poema breve de Luy, recopilado en la misma antología. El poema de la sección "Fragmentos y más poemas" que conforma el último de la serie del corpus propuesto tiene escrito lo siguiente: "Si el estado es virtuoso el hombre puede ser / libre" (cfr. anexo VI), dos versos que habilitan la comparación con el poema de un solo verso "Si va a morir gente, votemos quienes" (2009: 81). En la prótasis del poema del corpus (ubicado fuera del cuerpo central del libro), tenemos el verbo en presente del indicativo "es", que primó en varios otros poemas de la serie, mientras que en la apódosis vuelve a aparecer el tiempo presente del modo indicativo de "poder". La selección de tiempos y modos no es precisamente inocente si se considera el fuerte componente de apelación y orden profético-taxativo del yo lírico. $O$ dice las cosas como son, constata, remite a algo cierto, "indica"; o señala cómo serán, sin espacio a la duda, característica de algo que podríamos llamar "función profética".

En términos de organización métrica, y como última característica a tener en cuenta, los poemas del corpus se encuentran compuestos, en el escorzo textual, por versos irregulares, sin rima (con excepción de "La consciencia debiera ser obligatoria...", cfr. anexo II). Su agrupación presenta un tipo de regularidad, sin embargo. Tiende siempre a estrofas de tres versos, las cuales se constituyen por su función semántico-lógica. En el poema segundo de la serie, el ya citado "La consciencia...", la agrupación estrófica de tres versos coincide con un corte visual. Por función semántico-lógica, el primer terceto tiene el orden de una sentencia: parte de un dictum en el primer verso, con un corte fuerte marcado por la presencia del punto y coma, para avanzar, en el segundo verso, con la conjunción ilativa "luego", que da la apariencia de un razonamiento lógicoformal. Ese segundo verso tiene una función semántica aditiva: suma a lo propuesto en el primer verso, la "consciencia", otro elemento obligatorio, "la memoria". Finalmente, el terceto cierra con una conclusión, que también tiene el impacto energético del dictum de apertura: "la piedad es un error". La segunda estrofa fue analizada más arriba, ya que prima en ella el uso del modo imperativo. La estrofa no termina en una pausa fuerte, sino que lleva al tercer terceto por el cierre con una coma, produciendo una pausa. El segundo verso del tercer terceto vuelve a repetir el último verso del primer terceto, pero aquí matizado por un primer verso que sugiere la posibilidad de una diferencia de opinión a través de un condicional (“... si bien se ve...”). El último verso del tercer terceto, el cierre del poema, repite el primer verso, sólo que cambia el sustantivo: "consciencia" por "libertad". Se refuerza así la estructura en espejo que ya está dada por el ensamblaje de imagen y texto: el escorzo textual, en sí mismo, se encuentra trabajando una serie de repeticiones que marcan el ritmo del poema. La relación entre "consciencia", "libertad" y "memoria" como elementos opuestos a "piedad" también dejan en claro el tema central de la serie: la importancia de la apelación al otro-pueblo a partir de la indicación de un comportamiento que se debe llevar adelante. La necesidad, por parte del yo lírico, de establecer qué es lo que se debe hacer y cómo, algo que, dentro del conjunto de las figuras retóricas tradicionales, coincide con la parénesis. 


\section{El pueblo en pie de guerra: la parénesis} concreto del poema esté situado en un espacio de conflicto, de lucha, de diferencia que, muchas veces, toma la forma concreta de un encuentro bélico. La guerra, entonces, es uno de los temas que acompaña la parénesis. El trabajo de Ramón Alcalde "Lírica arcaica, elegía y parénesis" retoma esta vinculación, entendiendo que el llamado a la acción pública en el marco de una guerra respondía a necesidades coyunturales que también evidenciaban un rol social particular del poeta. Señala Alcalde en una nota al pie, hablando de la elegía, sí, pero como un componente más en la relación entre parénesis y guerra:

La elegía se desprende del mito; el poeta deja de ser el aedo depositario pasivo del pasado para convertirse en dirigente político o en sophós moral; se libera de la dependencia actual de la inspiración de las Musas y afirma su singularidad individual en cuanto miembro de una comunidad cívica articulada por valores y deberes suprapersonales. (1996: 41)

El contexto histórico de una elegía dominada por la parénesis implicaba la guerra y el uso de "un procedimiento retórico global" en las composiciones de poetas como Calino y Tirteo respondía a "las circunstancias históricas en que ambos poetiza(ron) (invasiones ciméricas en Asia Menor; guerras mesénicas en el Peloponeso)", en donde se enfrenta el poeta y su composición a "una misma problemática vital: la guerra y la conducta colectiva que frente a ella tienen que adoptar los miembros de la polis" (1996: 41-42).

"Pueblo", término presente en Luy para construir al interlocutor de los poemas, se carga a partir de esta lectura de un nuevo contenido: es un "pueblo" que se prepara al enfrentamiento. Un enfrentamiento que es leído como una amenaza de tipo interior: el enemigo no es externo, sino que responde a la identificación de una parte incorrecta, hostil, enemiga a un proyecto grupal, coherente. Por eso, la importancia de la "consciencia" y la "memoria" como herramientas de lucha: sirven para recordar e identificar a ese enemigo interno, con el que no se tiene que tener "piedad". La "obligatoriedad" de todas estas instancias forman parte del tipo de comportamiento que se debe adoptar en una situación de lucha y diferencia. Ese enemigo interno es la propia conformación estatal, el Estado, sin más: se genera así una distancia que rompe la identificación, podría decirse, "moderna", entre pueblo y Estado, y un intento de regreso a un modo de vinculación pre-estatal (¿o pos-estatal?). El cuarto verso del tercer poema del corpus trabajado, "La violencia es funcional al modelo'..." ( $c f r$. anexo III), lo dice claramente y, otra vez, bajo la estructura de una ilación de tipo consecutivo, lógico: "Luego, hay que atacar al sistema". Los "3 poderes" remiten como parte de una sinécdoque a lo estatal que se percibe como incorrecto, corrupto, algo que debe ser controlado (como lo pide el primer poema de la serie en función de los "bienes", cfr. anexo I) y cuyo control no puede quedar sólo en la revisión, sino que exige pasar a la acción, a la violencia abierta (algo que queda sugerido en el poema en donde "Lanata" es el interlocutor: "Cuando el documento ya no / documenta / ¿seguís investigando?", cfr. anexo IV). No podemos decir que la serie conforma un intento por proponer una forma de organización nueva diferente a la del "sistema". No hay momento propositivo, salvo por el llamado al enfrentamiento. El "deber cívico" es la guerra.

Cuadernos LIRICO, 20 | 2019 
¿Queda el yo lírico integrado al "pueblo" en esa lucha? El tono profético y hasta propio de un censor moral que tienen algunos versos construyen un yo no del todo integrado: bien se puede decir, a la vanguardia de lo que está señalando. Entendiendo, claro, en "vanguardia", su estricto significado bélico: la primera avanzada de soldados que van al choque, compuesta, generalmente, por los más valientes y jóvenes (y, a veces, más inexpertos). La disposición en espejo de los poemas que se detalló en el apartado anterior sirve para pensar esta oposición, ahora vista como repetición en la disposición en la página de estructuras que abarcan otras dimensiones (la semántica, la formal en términos morfosintácticos, etc.): no es sólo la diferencia entre el enemigo y el yo poético, sino también entre el yo poético y el "pueblo" al cual exhorta la que prima en la serie. Ese "pueblo" está también integrado por el "yo", pero sin síntesis que permita la absoluta coincidencia. Una diferencia mínima, pero esa diferencia entre uno y otro permite pensar a ese "yo" armado por los poemas como una subjetividad solitaria, que retoma, por un lado, el modo de construcción del escritor romántico estudiado por Benichou, mencionado en el primer apartado; y, por otra, la modalidad de un "poeta" que hace las veces de "orador" en tiempos de crisis, lo que recuerda el comentario de Albin Lesky en su Historia de la literatura griega que el propio Alcalde cita: "Calino y Tirteo entrañan la creación de aquella elegía política que se escuchó mientras la polis griega tuvo una vigorosa vida propia y el orador no había reemplazado aún al poeta" (1989: 145).

\section{Modos de identificación, modos de diferencia}

La parénesis como estrategia retórica global, la disposición de estructuras opositivas y en espejo, en abierta tensión, y el contenido semántico general de la serie determinan un tipo de procedimiento que recorre a toda la poesía de Luy: el llamado a un enfrentamiento violento como parte de una ética pública que parece perdida, pero que el yo lírico busca retomar, reescribir en su clave, directamente, construir. En función del contexto de producción de estos poemas, se podría pensar en un desplazamiento de cierto modo de construcción del "pueblo" del discurso político a la poesía, desplazamiento que coincide con un momento de crisis representativa que va desde 1999 hasta 2003 dentro del panorama político-histórico argentino, y que coincide con el fin del "menemato", el comienzo de la Alianza, la crisis del 2001 y el ascenso del kircherismo ${ }^{6}$ (fecha que coincide con dos de los libros de Luy más importantes, La vida en Córdoba, de 1999; y No le pidan peras a Cúper, de 2003). Tal como afirma Ernesto Laclau en La razón populista, el concepto de "pueblo" implica una diferencia de base entre conjuntos opuestos del cuerpo social y, al mismo tiempo, la constitución catacrética de una totalidad. El "pueblo" es un "nosotros" contra un "ellos", algo que actualiza en el terreno de la política efectiva una diferencia ontológica fundamental ${ }^{7}$. Chantal Mouffe, en Agonística, insiste sobre esta oposición al nombrar a la diferencia ontológica como "lo político" y a los modos de lidiar con esa diferencia a través de un sistema de representaciones partidarias como "la política". ¿De qué manera se puede recuperar este tipo de afirmación dentro del campo de la filosofía política para el análisis de poesía?

17 En el caso de la obra de Luy y, específicamente, en lo que se refiere a la serie analizada, se puede considerar que el modo de construcción de la voz del yo lírico, las operaciones aquí analizadas y el modo de recuperar productivamente un contexto sociopolítico (el 
de la crisis de representatividad de 1999-2003) podría implicar una insistencia de tipo poético-literaria en el corrimiento de "la política" como nivel de organización simbólico-representativa a "lo político", como la puesta en relieve de una diferencia ontológica de base que implica necesariamente el conflicto, en este caso, denominado "guerra". Pero eso no implica que el discurso literario-poético haya aparecido en ese momento para reemplazar un discurso ineficaz o imposible en otros términos. La literatura no "reemplaza" a "la política". Muy por el contrario, el hecho de que en la obra de Luy se retomen y aparezcan, una y otra vez, los mismos tópicos y hasta los mismos poemas, reubicados en nuevas antologías, repetidos, reescritos, muestran una repetición dentro de la obra que puede entenderse productivamente en función del problema de la consideración autónoma o posautónoma de la producción poética del presente ${ }^{10}$. La obra de Luy busca incidir políticamente al insistir de este modo, pero en la medida en que no ocupa un lugar dentro del discurso político, sino que sigue siendo leída como literatura, termina por reforzarse, por un lado, la diferencia entre el discurso literario y el político. Y, por otro, el lugar "solitario" de ese yo lírico construido. La parénesis se convierte, así, en el momento de mediación entre dos esferas específicas, separadas, que buscan superar su punto límite e ingresar en lo otro. La poesía de Luy apunta a lo político, intenta rebasar el límite autónomo, y tiene su momento de verdad en la medida en que realza el conflicto, "lo político", como base ontológica de todo presupuesto representativo. Pero pierde su vigencia en la medida en que no logra superar ese límite literario y permanece más acá: sigue siendo poesía, algo que deviene, en esta dialéctica no sintética que destacábamos en Adorno, tanto un logro como un terrible fracaso.

Si el kirchnerismo logró construir una nueva hegemonía a partir de prácticas discursivas determinadas que volvieron a poner en escena un tipo de representatividad de "la política", la permanente edición de antologías y, en algunos casos, el tratamiento estrictamente temático de algunos temas de coyuntura por parte de la poesía de Luy revela un vacío dentro de ese sistema de representación: la diferencia como lo emergente en todo vínculo representativo. Ese vínculo representativo busca, a través de operaciones retóricas, llenar esas distancias, alivianar, silenciar, controlar la diferencia. Pero, en tanto diferencia, sólo la literatura, lo "diferente" a "la política", puede convertirse en el espacio de "lo político", nombre de una diferencia ontológica imposible de acallar. Diferencia última que, siguiendo en alguna medida a Luy y a cierta filosofía política, puede llamarse de una manera por demás tajante: guerra civil.

\section{BIBLIOGRAPHY}

AAVV, Verbonautas. Acción poética, Buenos Aires, Eudeba, 1999.

Adorno Theodor, Teoría estética, tr. Fernando Riaza y Francisco Pérez Gutierrez, Madrid, Hyspamérica, 1984.

Alcalde Ramón, “Lírica arcaica, elegía y parénesis”, Estudios críticos de poética y política, Buenos Aires, Conjetural, 1996. 
Benichou Paul, La escuela del desencanto, tr. Alejandro Merlín, México, FCE, 2017.

Bogado, Fernando, "Crítica e imagen: lectura comparada de Aquí América Latina de Josefina Ludmer y Plan de operaciones de Vicente Luy", Perífrasis. Revista de literatura, teoría y crítica, número 17, vol. 9, enero-junio de 2018, Bogotá, Universidad de los Andes, p. 113-129.

De Diego José Luis (dir.), Editores y políticas editoriales en Argentina (1880-2010), Buenos Aires, FCE, 2014.

Deleuze Gilles y Félix Guattari, El Anti-Edipo. Capitalismo y esquizofrenia, tr. Francisco Monge, Buenos Aires, Paidós, 2010.

FNA, “Becas Creación 2018, Ganadores”, Web. Consultado el 1/12/2018.

Gago, Verónica, La razón neoliberal. Economías barrocas y pragmática popular, Buenos Aires, Tinta Limón, 2014.

Godio, Julio, Argentina: en la crisis está la solución. La crisis global desde las elecciones de octubre de 2001 hasta la asunción de Duhalde, Buenos Aires, Biblos, 2002.

Laclau Ernesto, La razón populista, tr. Soledad Laclau, Buenos Aires, FCE, 2011.

Lesky Albin, Historia de la literatura griega, tr. José María Díaz Regañón y Beatriz Romero, Madrid, Gredos, 1989.

Link Daniel, “Posfilología”, Suturas: Imágenes, escritura, vida, Buenos Aires: Eterna Cadencia, 2015. p. 117-135

Ludmer Josefina, Aquí América Latina. Una especulación, Buenos Aires, Eterna Cadencia, 2010.

Luy Vicente (1999), La vida en Córdoba, Córdoba, edición de autor.

--- (2000), Poesía moderna, s/d.

--- (2002), Aviones, s/d.

--- (2003), No le pidan peras a Cúper, Buenos Aires, edición de autor.

--- (2006), La sexualidad de Gabriela Sabatini, Buenos Aires, edición de autor.

--- (2007), Vicente habla al pueblo, Córdoba, La Creciente.

--- (2009), Poesía popular argentina, Buenos Aires, CILC.

--- (2011), ¡Qué campo ni campo!, Córdoba, Llanto de Mudo.

--- (2014), Plan de operaciones / La única manera de vivir a gusto es estando poseído, Buenos Aires, Crack-Up.

--- (2018) Caricatura de un enfermo de amor (1991), Buenos Aires, Años Luz.

Moscardi Matías, La máquina de hacer libritos. Poesía argentina y editoriales interdependientes en los noventa, Mar del Plata, Puente Aéreo, 2016.

Mouffe Chantal, Agonística. Pensar el mundo políticamente, tr. Soledad Laclau, Buenos Aires, FCE, 2014.

Schoijet Mauricio, La crisis argentina: los movimientos sociales y la democracia representativa, México, Plaza y Valdés, 2005. 


\section{NOTES}

1. La categoría de "editoriales independientes" posee diversas acepciones en función del estudio considerado. Para pensar el recorte que tomamos en el presente trabajo, cfr. De Diego 2014 y Moscardi 2016.

2. En la comunicación de los resultados de la convocatoria a Becas Creación del Fondo Nacional de las Artes correspondiente al año 2018, beca consistente en la entrega de $\$ 50.000$ dados con el fin de que el solicitante puede llevar a cabo un proyecto artístico en el marco de la disciplina elegida (artes escénicas, audiovisual, letras, etc.), Flavio Lo Presti resultó ganador con el proyecto "Enfermo de amor. Un retrato de Vicente Luy". La breve justificación del proyecto carga con toda la esperable retórica del "autor maldito", que nadie "leyó", y cuya "trágica" e "interesante" vida no le dejó alternativa otra que el suicidio. Leemos en la breve descripción del citado proyecto en la página del FNA (2018 Web): “Retrato literario (biografía) del poeta cordobés Vicente Luy, quien perdió a sus padres a los seis meses de edad, heredó una cuantiosa fortuna que gastó de manera inmoderada a lo largo de una vida llena de caprichos y, finalmente, terminó en una miseria triste que no pudo soportar y que lo condujo al suicidio".

3. Deleuze y Guattari critican la reinterpretación lacaniana del trabajo de Melanie Klein sobre los “objetos parciales", así como también la propia idea de Klein en torno a la relación entre objetos parciales y Objetos completos. Cfr. Deleuze y Guattari, 2010.

4. Queda por discutir, en trabajos posteriores, si ese ejercicio de reescritura es o no una "condición postmoderna" de la obra de Luy. Y si, bajo la condición de que estamos hablando de una "obra" que borra sus límites con respecto al concepto de "borrador", no estamos hablando, estrictamente, de un "archivo", tal como proponen los acercamientos de la posfilología hacia diversas materialidades textuales. (cfr. Link 2015: 117-135.).

5. Jorge Lanata (Mar del Plata, 1960). Periodista argentino de renombre, fundó el diario Página 12 y Crítica. En la actualidad, trabaja para el multimedio del Grupo Clarín.

6. Muchos son los estudios dedicados a esta "crisis representativa" y a los hechos históricos que dominaron lo que también ha sido llamado como crisis de una hegemonía y eventual reemplazo por otra. Cfr. Schoijet, 2005; Godio, 2002; Gago, 2014.

7. Inclusive en su caracterización del "pueblo" como término de suma importancia para su teoría política, Laclau entrevé la posibilidad grupal de encontrar algún tipo de orden: ¿puede la poesía llenar un vacío político? Leemos en Laclau: “(...) cuando la gente se enfrenta a una situación de anomia radical, la necesidad de alguna clase de orden se vuelve más importante que el orden óntico que permita superarla" (subrayado en el original, 2011: 116). Para una caracterización completa del uso de Laclau del término "pueblo", cfr. pp. 89-216, especialmente pp. 91-161.

8. Leemos en Mouffe: "Lo político" se refiere a esta dimensión de antagonismo que puede adoptar diversas formas y puede surgir en diversas relaciones sociales. Es una dimensión que nunca podrá ser erradicada. Por otro lado, "la política" se refiere al conjunto de prácticas, discursos e instituciones que busca establecer un determinado orden y organizar la existencia humana en condiciones que siempre son potencialmente conflictivas, ya que están afectadas por la dimensión de "lo político" (2014: 22).

9. Poemas posteriores de Luy dan el nombre efectivo al tipo de conflicto que aparece en toda su producción. En Plan de operaciones, la primera mitad de su, hasta ahora, único libro póstumo, puede leerse:

Vicente habla con su discípulo

[...]

D- ¿Qué busca el libro?

V- Trata de blanquear la guerra. 
No estamos de acuerdo con esto, y vamos a hacer nuestra movida.-

(2014: 20).

10. Trabajé este tema en un artículo dedicado a revisar el concepto de "posautonomía" de Josefina Ludmer en relación a la poesía de Vicente Luy. Cfr. Bogado, 2018 y Ludmer, 2010.

\section{ABSTRACTS}

The work of Vicente Federico Luy (1951-2012) evidences a formal internal tension that proposes, as poetry, to intervene in Argentine politics. Through the study of a series of pieces linked to the guiding phrase "Vicente habla al pueblo" ("Vicente speaks to the people"), this article analyzes the presence in these poems of the parenesis as a global rhetorical figure, the importance of a mirror structure that links image and text, and the use of concepts such as "people" and "war" as thematic instances; all relevant moments to understand the ways of a relationship mediated between two extremes: literature and society.

La obra de Vicente Federico Luy (1951-2012) evidencia una tensión formal interna en la medida en que propone, en tanto poesía, intervenir en la política argentina. A través del estudio de una serie de piezas vinculadas a la frase rectora "Vicente habla al pueblo", este artículo analiza la presencia en esos poemas de la parénesis como figura retórica global, la importancia de una estructura en espejo que vincula imagen y texto, y la utilización de conceptos como "pueblo" y "guerra" como instancias temáticas; todos momentos relevantes para entender los modos de una relación mediada entre dos extremos: literatura y sociedad.

L'œuvre de Vicente Federico Luy (1951-2012) met en évidence une tension interne formelle puisqu'elle propose, en tant que poésie, d'intervenir dans la politique argentine. À travers l'étude d'une série d'oeuvres liées à la phrase directrice "Vicente habla al pueblo" ("Vicente parle au peuple"), cet article analyse la présence dans ces poèmes de la parénèse en tant que figure rhétorique globale, l'importance d'une structure en miroir reliant image et texte, et l'utilisation de concepts tels que "peuple" et "guerre" en tant qu'instances thématiques; ce sont des moments pertinents pour comprendre les modalités d'une relation médiatisée entre deux extrêmes: la littérature et la société.

INDEX

Palabras claves: guerra, pueblo, parénesis, política, poesía argentina del siglo XXI.

Keywords: war, people, parenesis, politics, Argentine poetry of the 21th Century.

Mots-clés: guerre, peuple, parénèse, politique, poésie argentine du 21 ème siècle.

\section{AUTHOR}

\section{FERNANDO BOGADO}

Universidad de Buenos Aires - Conicet

fernandobogado@outlook.com 